\title{
1 Application of transposon-insertion sequencing to determine gene essentiality in the acetogen
}

\section{Clostridium autoethanogenum}

3 Craig Woods ${ }^{1}$, Christopher M. Humphreys ${ }^{1}$, Claudio Tomi-Andrino ${ }^{1,2,3}$, Anne M. Henstra ${ }^{1}$,

4 Michael Köpke ${ }^{4}$, Sean D. Simpson ${ }^{4}$, Klaus Winzer ${ }^{1}$, Nigel P. Minton ${ }^{1,5}$

$5{ }^{1}$ Clostridia Research Group, BBSRC/EPSRC Synthetic Biology Research Centre (SBRC),

6 Biodiscovery Institute, School of Life Sciences, The University of Nottingham, Nottingham,

$7 \quad$ NG7 2RD, UK

$8{ }^{2}$ Centre for Analytical Bioscience, Advanced Materials and Healthcare Technologies

9 Division, School of Pharmacy, The University of Nottingham, Nottingham, NG7 2RD, UK

$10{ }^{3}$ BBSRC/EPSRC Synthetic Biology Research Centre (SBRC), School of Mathematical

11 Sciences, The University of Nottingham, Nottingham, NG7 2RD, UK

$12 \quad{ }^{4}$ LanzaTech Inc., 8045 Lamon Avenue, Suite 400, Skokie, IL, USA

$13 \quad{ }^{5}$ NIHR Nottingham Biomedical Research Centre, Nottingham University Hospitals NHS

14 Trust and the University of Nottingham, Nottingham, NG7 2RD, UK.

16 Corresponding author: Professor Nigel Minton (email: nigel.minton@ nottingham.ac.uk)

19 Running Title: Transposon-insertion sequencing in an acetogen 


\section{ABSTRACT}

21 The majority of the genes present in bacterial genomes remain poorly characterised with up to one third of those that are protein encoding having no definitive function. Transposon insertion sequencing represents a high-throughput technique that can help rectify this deficiency. The technology, however, can only be realistically applied to easily transformable species leaving those with low DNA-transfer rates out of reach. Here we have developed a number of approaches that overcome this barrier in the autotrophic species Clostridium autoethanogenum using a mariner-based transposon system. The inherent instability of such systems in the Escherichia coli conjugation donor due to transposition events was counteracted through the incorporation of a conditionally lethal $\operatorname{codA}$ marker on the plasmid backbone. Relatively low frequencies of transformation of the plasmid into $C$. autoethanogenum were circumvented through the use of a plasmid that is conditional for replication coupled with the routine implementation of an Illumina library preparation protocol that eliminates plasmid-based reads. A transposon library was then used to determine the essential genes needed for growth using carbon monoxide as a sole carbon and energy source.

IMPORTANCE. Although microbial genome sequences are relatively easily determined, assigning gene function remains a bottleneck. Consequently, relatively few genes are well characterised, leaving the function of many as either hypothetical or entirely unknown. Highthroughput, transposon sequencing can help remedy this deficiency, but is generally only applicable to microbes with efficient DNA-transfer procedures. These exclude many microorganisms of importance to humankind either as agents of disease or as industrial

42 process organisms. Here we developed approaches to facilitate transposon-insertion sequencing in the acetogen Clostridium autoethanogenum, a chassis being exploited to 
44 convert single-carbon waste gases, $\mathrm{CO}$ and $\mathrm{CO}_{2}$, into chemicals and fuels at an industrial

45

46

47

scale. This allowed the determination of gene essentiality under heterotrophic and autotrophic growth providing insights into the utilisation of $\mathrm{CO}$ as a sole carbon and energy source. The strategies implemented are translatable and will allow others to apply transposon-insertion sequencing to other microbes where DNA-transfer has until now represented a barrier to progress.

\section{INTRODUCTION}

Although microbial genome sequences are relatively easily determined, assigning gene function remains a bottleneck. Consequently, relatively few genes are well characterised, leaving the function of many as either hypothetical or entirely unknown. Thus, even the Syn 3.0 minimal genome retains 149 genes (32\%) of unknown function ${ }^{1}$. A greater understanding of gene functionality can be gleaned through the deployment of high throughput transposon sequencing. This technique is characterised by the simultaneous Illumina sequencing of the site of transposon insertion in pooled mutant libraries using a sequencing primer specific to the transposon-chromosomal junction. If the library consists of a sufficiently high number of unique insertions then the required gene set for the growth conditions used can be inferred since unrepresented or underrepresented genes are likely to be essential. There are several names for this type of approach, including the first four all published in 2009: TraDIS ${ }^{2}$, HITS ${ }^{3}$, Tn-Seq and INSeq ${ }^{4}$. All of these techniques aim to identify the position and quantity of transposon mutants and are collectively referred to as Transposon insertion sequencing (TIS) ${ }^{5}$.

The deployment of TIS typically is largely dependent on high frequency DNA transfer. This excludes its application to many microbial species. Anaerobic bacteria, and in particular 
members of the genus Clostridium, are of both medical and industrial importance but generally display low rates of DNA transfer. This has limited the exploitation of TIS in this grouping where to date TIS has only been applied ${ }^{6}$ to the pathogen Clostridioides difficile (formerly Clostridium difficile). One group of bacteria with increasing importance are the anaerobic acetogens, typified by Clostridium autoethanogenum. Acetogens possess the Wood-Ljungdahl pathway (WLP), or reductive acetyl-CoA pathway, which allows the fixation of $\mathrm{CO}$ and $\mathrm{CO}_{2}{ }^{7}$. Suggested to be the earliest autotrophic pathway ${ }^{8}$, it is the most energy efficient of the seven known carbon fixation pathway since it conserves energy while all others require its input ${ }^{9}$. Reducing equivalents needed for metabolic processes are obtained either from $\mathrm{H}_{2}$ or $\mathrm{CO}$ using hydrogenases or $\mathrm{CO}$ dehydrogenase (CODH), respectively. Carbon is fixed via the Eastern branch of the pathway where, through a series of cobalamin and tetrahydrofolate-dependent reactions, $\mathrm{CO}_{2}$ is reduced to a methyl group. The methyl group from the Eastern branch is then combined with CO to form acetyl-CoA which is the root of subsequent anabolic reactions ${ }^{10-13}$.

While the majority of acetogens synthesize acetate as the sole fermentation product some, typified by $C$. autoethanogenum, naturally produce industrially relevant compounds as 2-3 butanediol and ethanol, the latter on a commercial scale ${ }^{14}$. Commercial efforts to extend the product range further are ongoing with isopropanol being a notable example ${ }^{15}$. $C$. autoethanogenum is one of the best understood autotrophic acetogens with a manually annotated genome ${ }^{16,17}$ and has been subjected to transcriptomic and proteomic analysis ${ }^{18}$.

In the current study we sought to maximise the benefit of available $C$. autoethanogenum genome data through implementation of TIS. However, as DNA transfer into $C$. autoethanogenum is only possible at relatively low frequencies, a number of essential modifications to the procedure were required. Specifically, the use of a conditional replicon 
and an inducible orthogonal expression system to control production of transposase allowed the controlled generation of a large mutant library from a small number of initial transconjugant colonies. Additionally, the incorporation of I-SceI recognition sequences into the delivery vehicle provided a mechanism to eliminate those mini-transposon sequences still present on autonomous copies of the plasmid during the transposon mutant library preparations stage. These adaptations have allowed a thorough genetic analysis of the WLP in required for growth on $\mathrm{CO}$ as a sole carbon and energy source.

\section{RESULTS}

102 Control of transposition. A fundamental requirement of an effective transposon-delivery 103 system is that transposition should preferentially take place in the target strain and not in the

104 donor strain. This may be achieved through the use of an orthogonal expression system in which the promoter controlling production of the transposase is recognised by transcriptional factors that are only present in the target microbe. A previously described clostridial system promoters it recognises, the $\mathrm{P}_{t c d B}$ promoter of the toxin $\mathrm{B}$ gene $(t c d B)$. By generating a derivative of Clostridium acetobutylicum in which the TcdR-encoding $t c d R$ gene was inserted into the genome at the pyrE locus, any subsequently introduced gene that was placed under the control of the $\mathrm{P}_{t c d B}$ promoter is expressed. In this example, the gene placed under the control of this orthogonal system was the mariner transposase gene ${ }^{19}$. 


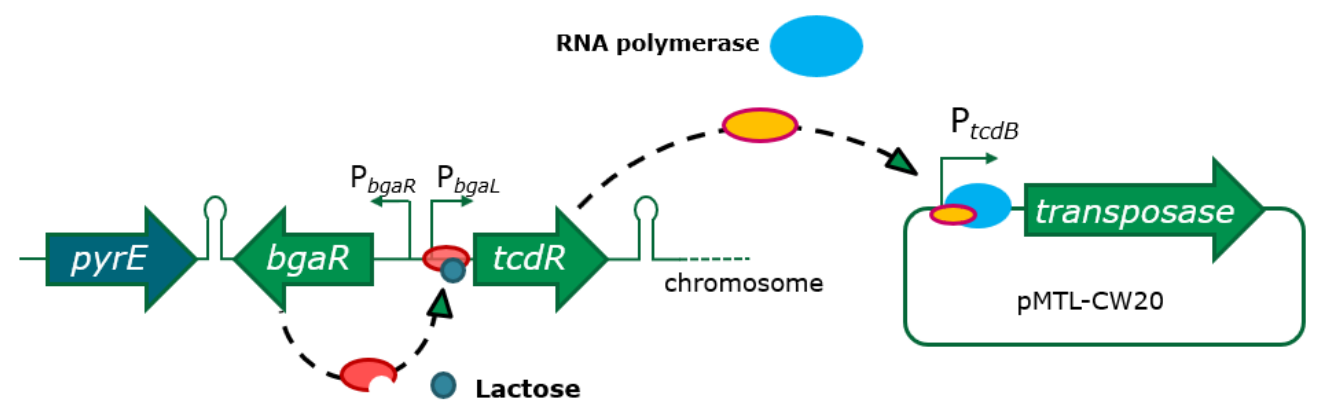

Figure 1. TcdR-mediated orthogonal expression. In C. autoethanogenum $\mathrm{C} 24$, tcdR is under

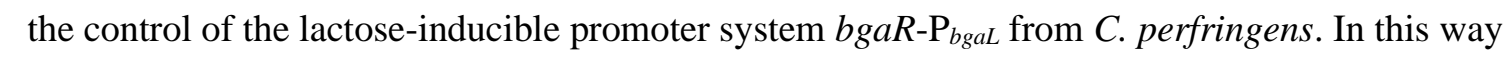
the $\mathrm{P}_{t c d B}$ promoter can be induced indirectly via the inducible expression of $t c d R$ from the chromosome.

For exploitation in C. autoethanogenum, further control was engineered into the system by placing expression of $t c d R$ (Fig. 1) under the control of a lactose inducible promoter $P_{b g a L}$ previously shown to be functional in the closely related Clostridium ljungdahlii ${ }^{20}$.

Accordingly, the $P_{b g a L}$ promoter, together with the necessary $b g a R$ which encodes a transcriptional regulator, was positioned $5^{\prime}$ to the $t c d R$ gene and the DNA module created (bgaR-P $\left.\mathrm{P}_{b g a L}:: t c d R\right)$ integrated into the $C$. autoethanogenum chromosome at the pyrE locus using ACE (Allele Coupled Exchange) ${ }^{20-23}$. This involved restoring a uracil-requiring $\Delta p y r E$ mutant strain to prototrophy concomitant with genomic insertion of $b g a R-\mathrm{P}_{g a L}:: t c d R$ using the ACE plasmid pMTL-CH20lactcdR. Successful mutant generation was confirmed by PCR analysis and Sanger sequencing of the amplified DNA and the resulting strain was termed $C$. autoethanogenum $\mathrm{C} 24$.

To confirm that TcdR production could be controlled by the addition of exogenous lactose in strain $\mathrm{C} 24$, the Clostridium perfringens catP reporter gene encoding a chloramphenicol acetyltransferase (CAT) was cloned downstream of the $\mathrm{P}_{t c d B}$ promoter on an appropriate clostridial shuttle vector. Regulation of the reporter gene was shown to be dependent on the addition of the lactose inducer (Figure 2). We therefore chose to use this expression system to 


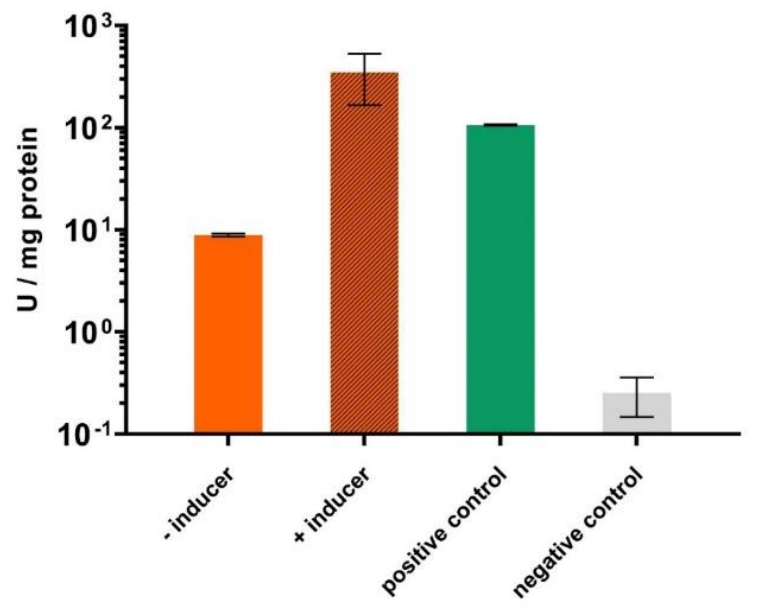

Figure 2. Chloramphenicol acetyl transferase (CAT) assay of lactose-inducible orthogonal system. Expression from $\mathrm{P}_{t c d B}$ was quantified using a CAT assay. Three plasmids were conjugated into $C$. autoethanogenum $P_{b g a L \_} t c d R(C$. autoethanogenum $\mathrm{C} 24)$ with each plasmid harbouring catP under the control of either $\mathrm{P}_{t c d B}, \mathrm{P}_{t h l}$ (positive control), or no promoter (negative control). The strain harbouring the $\mathrm{P}_{t c d B}$ plasmid was tested with and without the addition of 10 $\mathrm{mM}$ lactose while the remaining plasmids were tested without lactose. The data shown is the result of biological triplicates with error bars showing the standard deviation.

A second feature of pMTL-CW20 designed to control unwanted transposition was based on the provision of a promoter-less copy of the E. coli $\operatorname{codA}$ gene encoding cytosine deaminase to prevent premature transposition in the donor strain. Use of the previously described transposon-delivery vector pMTL-YZ14 ${ }^{24}$ was characterised by inconsistent frequencies of transfer to the clostridial recipient and/or to variation in the effectiveness with which transposon mutants were generated once in C. autoethanogenum. These inconsistencies appeared to correlate with spontaneous plasmid rearrangements in the donor, as evidenced by unexpected DNA fragment profiles on agarose gels of diagnostic digests of the isolated plasmid DNA (Fig. 3). This was assumed to be due to transposition of the mini transposon from pMTL-YZ14 while in E. coli either into the genome or, as transposition into closed 
circular autonomous plasmids is preferred, into alternative positions in the vector backbone. maintenance or ability to transfer. Similar instabilities have been noted elsewhere ${ }^{25}$.

Cytosine deaminase catalyses conversion of 5-fluorocytosine (5-FC) to the toxic product 5fluorouracil (5-FU) which ultimately blocks DNA and protein synthesis. On the plasmid pMTL-CW20, $\operatorname{codA}$ is separated from its $\mathrm{P}_{t h l}$ promoter (derived from the thiolase gene of Clostridium acetobutylicum) by the catP mini-transposon. Excision of the mini-transposon as a consequence of its transposition leads to expression of $\operatorname{cod} A$, a lethal event in the presence of exogenously supplied 5-FC. The addition of this feature to pMTL-CW20 improved the reproducibility with which the plasmid was transferred to $C$. autoethanogenum and appeared to prevent the occurrence of plasmid rearrangements (Fig. 3).

Removal of plasmid-based reads. Suicide vectors are inappropriate for $C$. autoethanogenum since the low DNA-transfer rate means an unfeasible number of conjugations would need to be performed with a preliminary experiment resulting in five mutants from three conjugations. To compensate for this, a conditional replicon was utilised which has been described previously ${ }^{24}$. To further remove any residual plasmid from the sequencing library I-SceI recognition sites were incorporated into pMTL-CW20 which provided a mechanism

172 for removal of plasmid reads at the sequencing library stage. After adapter-ligation an I-SceI restriction is used to cleave the site between the adapter and the library primer binding site on the transposon, making those fragments originating from plasmid DNA unsuitable templates

175 for the subsequent PCR amplification step as described in a similar strategy ${ }^{26}$. In the initial 176 transposon library grown on rich medium, $0.2 \%$ of reads mapped to the transposon-delivery 177 plasmid. This compares favourably with a study on Clostridiodes difficile which used a 
(a)

PMTL-CW20

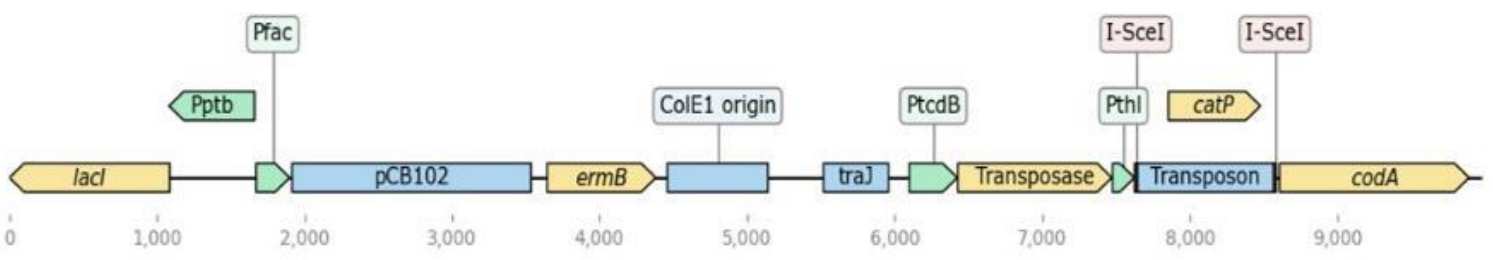

(b)

(c)

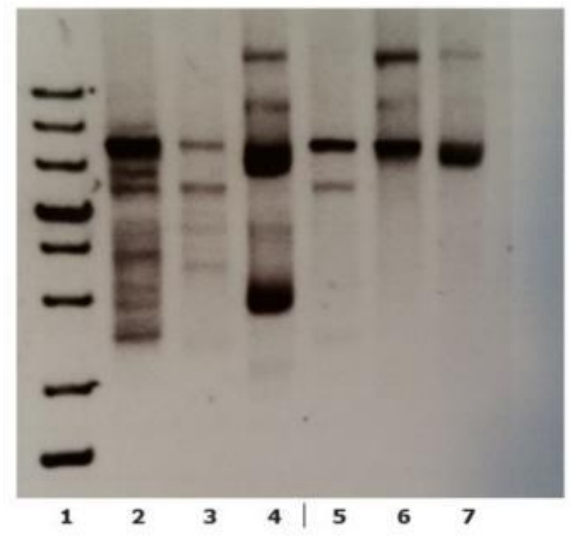

Figure 3. Transposon delivery plasmid pMTL-CW20. A) pMTL-CW20 is based on the pMTL-YZ14 plasmid described in ${ }^{24}$ using components from the plasmid modular transfer series outlined in ${ }^{27}$ as well as the $\operatorname{codA}$ from E. coli and I-SceI recognition sites. Replication occurs in E. coli via the pUC ColE1 origin of replication and the plasmid can be transferred to clostridial recipients using the oriT from RK2 ${ }^{28}$. In clostridial hosts the plasmid is conditionally replicative where the presence of IPTG is the non-permissive condition. Transposition is achieved via a hyperactive Himar1 variant ${ }^{29}$ which mobilises a mini-transposon containing the catP gene which confers chloramphenicol and thiamphenicol resistance. B) A verified pMTL-YZ14 plasmid was used to transform E. coli Top10 and transformant colonies used to inoculate overnight cultures. Plasmids prepared from overnight cultures were extracted and treated with SbfI. Movement of the transposon into various other parts of the vector was found to have occurred $(2,4,6$, and 7$)$ while only lanes 3 and 5 exhibited the expected band pattern. ThermoFisher $1 \mathrm{~kb}+$ plus ladder is in lane $1 \mathrm{C})$ An analogous procedure using EcoRV was later followed using pMTL-CW20 instead of pMTL-YZ14. In this case all six plasmids exhibited the expected band pattern (lanes 2-7) with ThermoFisher $1 \mathrm{~kb}+$ ladder in lanes 1 and 8 . 
$200 \mathrm{~mL}$ of rich medium (YTF) supplemented with thiamphenicol and IPTG. After $24 \mathrm{~h}$ of growth, genomic DNA was extracted from this culture and $-80^{\circ} \mathrm{C}$ freezer stocks were made.

This first genomic DNA extraction was used to determine the required gene set for growth on rich media, 100,065 unique insertion sites were found from this sample. Subsequently the freezer stocks were used to restore the mutant pool into a defined medium (PETC) supplemented with pyruvate as the carbon source. The PETC culture was used to inoculate a 1.5 L bioreactor containing fermentation medium which lacked a carbon source. The sole carbon and energy source after the inoculation was provided by $\mathrm{CO}$ gas sparged into the bioreactor with a gradual increase of CO. The pyruvate was quickly used up as shown by HPLC data (Supplementary Table S3) and C. autoethanogenum instead relied upon fixation of CO. The PETC medium provided no supplementary amino acids and instead relied on the native biosynthesis pathways of $C$. autoethanogenum. Vitamin requirements were met via the addition of Wolfes' vitamin solution.

Samples for HPLC analysis of metabolites and for possible genomic DNA extraction and TIS analysis were taken on a daily basis. Samples from 72, 144, 168, 336, and $360 \mathrm{~h}$ of growth were used for sequencing. These sequencing data were used to determine the required gene set for growth using CO in a defined medium. Ultimately, the samples from $336 \mathrm{~h}$ and $360 \mathrm{~h}$ were used to determine the gene set required for growth on $\mathrm{CO}$, these represent the endpoint of the reactor fed batch culture. The reactor endpoint was sequenced revealing 66,524 unique insertion sites.

Functions of essential genes in heterotrophic compared with autotrophic conditions. The functions of candidate essential genes for growth in rich medium and minimal medium with $\mathrm{CO}$ as the carbon and energy source were compared using the KEGG database as summarised in Table 1. There were 439 genes $(11 \%)$ identified as candidate essential genes out of a total 
of 4059 genes in the genome for heterotrophic growth on the rich medium YTF where fructose and yeast extract serves as a carbon and energy source (Supplementary Table S1). processes such transcription, translation, DNA replication and cell division are common in the rich media essential gene list. Eighteen of the twenty common amino acids have clearly annotated tRNA synthetases which appear essential except for tyrosine and asparagine. Tyrosine appears to exhibit redundancy via CLAU_1290 (tyrZ) and CLAU_1635. There is only one annotated asparagine tRNA synthetase $(\operatorname{asn} B)$ but it seems likely that there is another present (CLAU_2687) and that together they provide functional redundancy meaning that both genes are found to be non-essential. CLAU_2687 is currently annotated as a tRNA synthetase class II but is most likely to be an asparagine-specific tRNA synthetase. Another explanation for the non-essential status of the asparagine tRNA synthetase could be that $C$. entirely lack an asparagine tRNA synthetase. These taxa rely on a non-discriminating aspartic acid tRNA synthetase followed by an amidotransferase to generate asparagine-tRNAs ${ }^{30}$.

237 The candidate essential gene list for rich medium calls into question several of the annotations in the C. autoethanogenum genome. For instance, CLAU_0265 which is annotated as a small acid-soluble spore protein is required on rich medium despite $C$. autoethanogenum $\mathrm{C} 24$ never having been observed to sporulate. In addition, sporulation

241 should never have been required in the library preparation process. The gene must, therefore,

242 have an additional or alternative role. Much functional genomics work has yet to be

243 performed on $C$. autoethanogenum since there are 44 rich medium essential genes annotated 244 as hypothetical proteins (Supplementary Table S1). 


\begin{tabular}{|l|c|c|} 
Function & Rich & Minimal Medium + CO \\
\hline Metabolic Pathways & 98 & 156 \\
\hline Ribosome & 38 & 42 \\
\hline Microbial metabolism in diverse environments & 33 & 41 \\
\hline Carbon metabolism & 25 & 30 \\
\hline Aminoacyl-tRNA biosynthesis & 21 & 23 \\
\hline Biosynthesis of amino acids & 18 & 36 \\
\hline Hypothetical & 13 & 24 \\
\hline Peptidoglycan biosynthesis & 11 & 12 \\
\hline Pyruvate metabolism & 9 & 11 \\
\hline Carbon fixation pathways in prokaryotes & 9 & 13 \\
\hline DNA replication & 9 & 11 \\
\hline Amino sugar and nucleotide sugar metabolism & 9 & 9 \\
\hline Oxidative phosphorylation & 7 & 10 \\
\hline Fatty acid metabolism & 6 & 7 \\
\hline Fatty acid biosynthesis & 6 & 7 \\
\hline Propanoate metabolism & 6 & 7 \\
\hline Quorum sensing & 5 & 7 \\
\hline Protein export & 5 & 9 \\
\hline Purine metabolism & 5 & 14 \\
\hline Bacterial secretion system & 5 & 9 \\
\hline RNA degradation & 5 & 7 \\
\hline RNA polymerase & 4 & 4 \\
\hline Pyrimidine metabolism & 4 & 10 \\
\hline Citrate cycle (TCA cycle) & 3 & 7 \\
\hline Phosphotransferase system (PTS) & 2 & 2 \\
\hline Folate biosynthesis & 2 & 5 \\
\hline Biotin metabolism & 1 & 2 \\
\hline Flagellar assembly & 1 & 1 \\
\hline Bacterial chemotaxis & 1 & 2 \\
\hline Tato & 9 & \\
\hline
\end{tabular}

246 Table 1. Functions of essential genes. Number of C. autoethanogenum essential genes for

247 various KEGG functional categories on the rich medium YTF and the minimum fermentation

248 medium with $\mathrm{CO}$ as a carbon and energy source

249 In total, 758 genes (19\% of the genome) were predicted to be required for autotrophic growth

250 by the endpoint of the CO-fed reactor (Supplementary Table S1). This includes all of the

251 'core' gene set which were also required on rich medium and all of the genes required to

252 grow on minimal medium lacking amino acids. The core gene set was predicted to be

253 comprised of 439 genes. This means that 319 genes are likely to be required for the synthesis

254 of all amino acids and utilisation of $\mathrm{CO}$ as a carbon and energy source. As vitamins were

255 provided, their biosynthetic pathways were not expected to be represented, similarly nitrogen 
and sulphur were supplied in the medium. All genes and their predicted essentiality status in deducing condition-specific genes, but the data is nevertheless extremely informative.

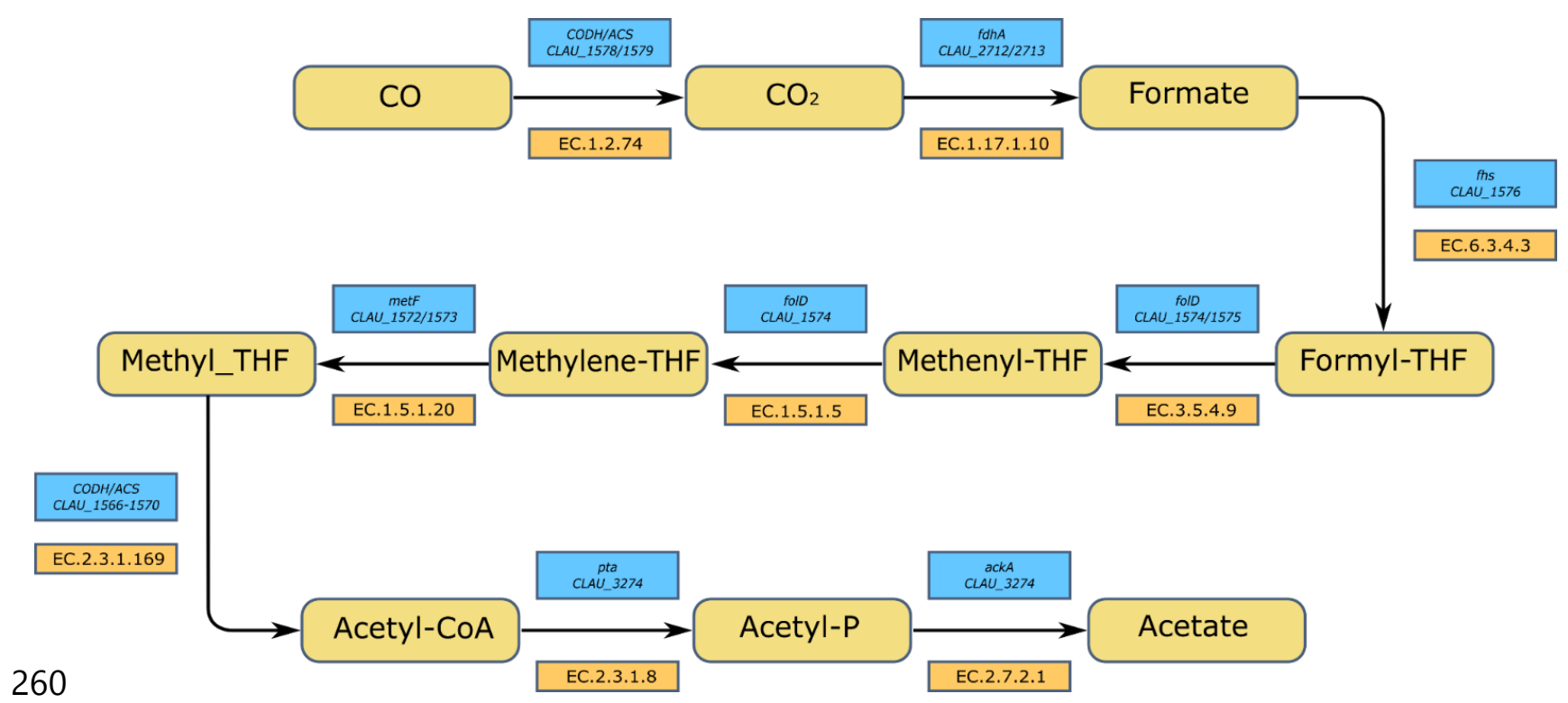

Figure 4. Essential Genes of the Wood-Ljungdahl pathway. Route from CO to acetate showing the expected gene/locus tag for each step. Each of the locus tags listed was required for growth on $\mathrm{CO}$.

264 Essential genes of the Wood-Ljungdahl (WLP) pathway. In order to grow using CO as a

265 sole carbon and energy source it is necessary for $C$. autoethanogenum to use two molecules of $\mathrm{CO}$ to form one molecule of acetyl-CoA. Acetyl- CoA consists of a methyl group, a

Eastern branch of the WLP before being combined with another CO molecule and CoA by

270 the acetyl-CoA synthase (ACS). It was therefore expected that all the genes involved in the

271 WLP would be required for growth on CO. A complete WLP was indeed found in the list of 
273 heterotrophic growth despite the fact that it is utilised during heterotrophic growth to fix

$274 \mathrm{CO}_{2}$ released during glycolysis using the reducing equivalents generated by glycolysis ${ }^{23}$.

Insertions in the rnf complex operon.
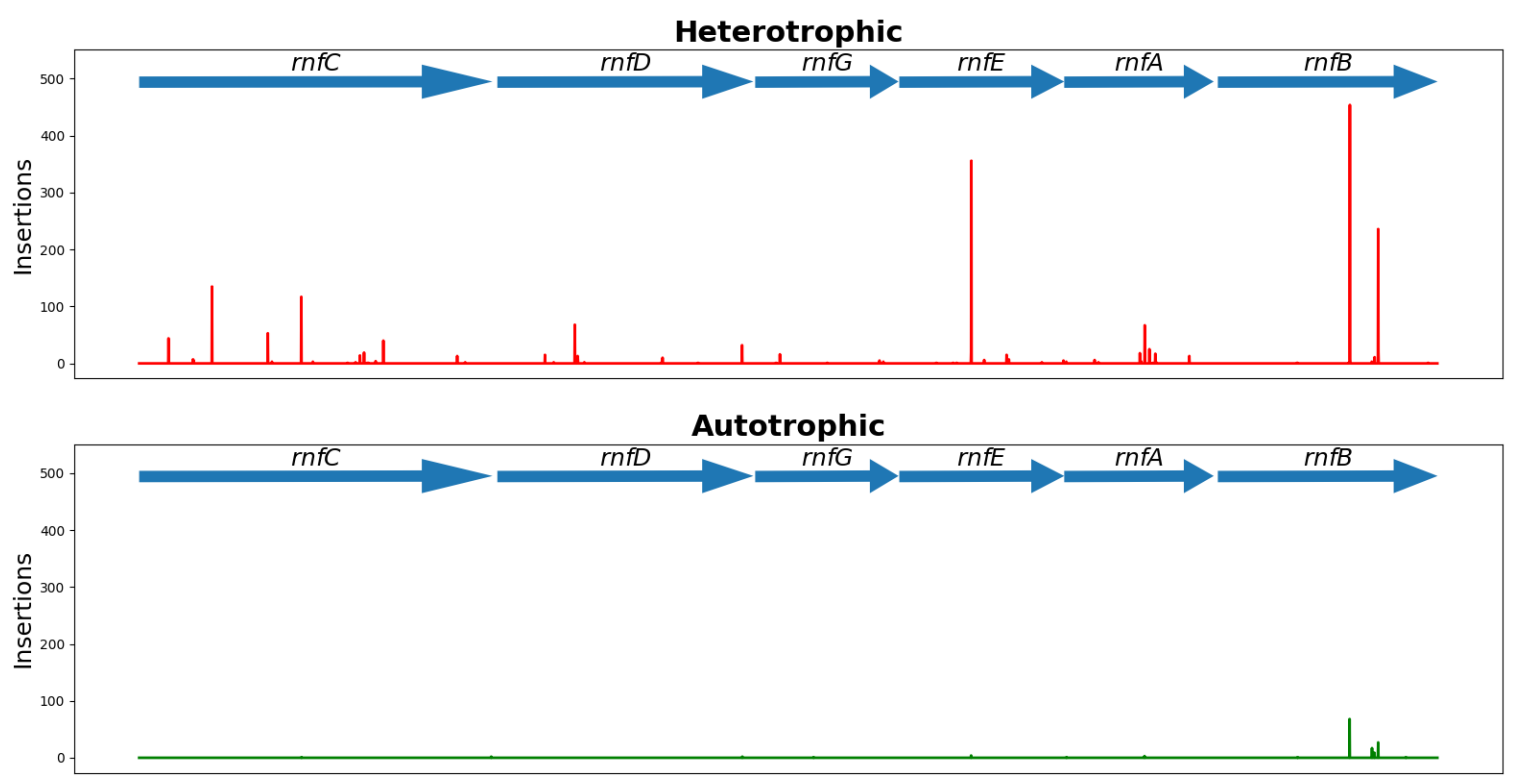

27

276

277

278

279

280

281

282

Figure 5: Insertions in the Rnf complex region. Number of reads detected along the genomic region encoding the Rnf complex for heterotrophic and autotrophic conditions. Insertions are relatively abundant in heterotrophic conditions implying importance for the complex under autotrophic conditions.

To generate ATP, C. autoethanogenum is reliant on generating a transmembrane electrochemical gradient via the intrinsically important ${ }^{31} \mathrm{Rnf}$ complex and a membrane integral ATP synthase ${ }^{18,32}$. The Rnf complex of $C$. autoethanogenum is encoded by the region CLAU_3144-CLAU_3149. A comparison of the insertion sites found in heterotrophic and autotrophic conditions for this region is shown in Fig. 5. With exception of $r n f B$, all of the encompassed genes were found to be essential for growth on CO (Fig.5), confirming previous observations that inactivation of these genes in either C. ljungdahlii and Acetobacterium woodii curtailed growth on $\mathrm{H}_{2}+\mathrm{CO}_{2}{ }^{33,34}$. Despite rnfB being above the insertion index threshold for essentiality on $\mathrm{CO}$, it is significantly under-represented when the data obtained from cells grown on pyruvate is compared with $\mathrm{CO}\left(\log _{2}\right.$ fold change $=-2.86$, 
$\mathrm{p}$-value $=1.16 \mathrm{E}-11)$. It may be the case that $r n f B$ encodes a non-essential component of the complex which aids functionality but is not required for it. In Methanogens RnfB has been characterized as an entry point for electrons to the Rnf complex ${ }^{35}$.

The importance of $\mathbf{N f n}$ for autotrophic growth. In order to further verify the calling of gene essentially under specific conditions using our parameters, a candidate gene was selected for directed CRISPR mutagenesis. The $n f n$ gene (CLAU_1539) encodes an electronbifurcating ferredoxin-dependent transhydrogenase, responsible for the production of NADPH from NADH and $\mathrm{Fd}^{2-}$, thus recycling NAD+. Our TIS data analysis found that the $n f n$ gene was non-essential when $C$. autoethanogenum was grown on rich medium or when grown on minimal medium with pyruvate, but when autotrophic conditions were used the gene was essential. This suggested that a directed CRISPR knockout mutant should be obtainable while the culture is grown under heterotrophic conditions but should fail to survive when transferred to autotrophic conditions. A CRISPR in-frame deletion mutant of $n f n(\Delta n f n)$ was created which was viable on rich media, and on minimal medium with pyruvate as a carbon source, but was unable to grow when $\mathrm{CO}$ was the sole carbon and energy source.

Initially the $\Delta n f n$ strain was characterised in serum bottles, using minimal PETC media and either $10 \mathrm{mM}$ of sodium pyruvate, or 1 bar of $\mathrm{CO}$ in the headspace, as the carbon and energy source. Serum bottles were inoculated with $1 \mathrm{ml}$ (1:50 inoculum) of a late exponential culture grown in the anaerobic cabinet on minimal media with fructose as a carbon source. The cultures grown on pyruvate grew similarly to the wild type control, however, no evidence of

311 growth was evident when $\mathrm{CO}$ was used instead of pyruvate as the carbon and energy source.

312 This inability to utilise $\mathrm{CO}$ as a carbon and energy source was further demonstrated on a

313 larger scale using a fed-batch CSTR experiment, whereby a 1.5 L culture was inoculated with 
$314150 \mathrm{ml}$ of an early exponential culture grown on minimal media and pyruvate. The $\mathrm{pH}$ was

315 controlled with $\mathrm{NaOH}$ and $\mathrm{H}_{2} \mathrm{SO}_{4}$, and sparged through continual addition of nitrogen at a

316 rate of $60 \mathrm{ml} / \mathrm{min}$. At the time of inoculation $5 \mathrm{mM}$ of sodium pyruvate was added to the

317 culture. Once an $\mathrm{OD}_{600}$ of approximately 0.3 had been reached, $\mathrm{CO}$ was introduced at a rate

318 of $10 \mathrm{ml} / \mathrm{min}$. In the case of the wild type culture, the strain was able to adapt to the $\mathrm{CO}$

319 carbon and energy source and after $48 \mathrm{~h}$ the OD continued to increase after the pyruvate had

320 been depleted. In the case of the $n f n$ mutant, the culture was not able to adapt to utilising $\mathrm{CO}$,

321 and the optical density rapidly declined following depletion of the pyruvate.

322 Assessment of metabolic modelling-derived gene essentiality. Experimentally confirmed

323 gene essentiality for growth on minimal medium supplemented with $\mathrm{CO}$ was compared

against the predicted essentiality calculated from a metabolic model of $C$. autoethanogenum

(MCC), a robust metric ranging from -1 to +1 used to evaluate binary classifications (such as essential or non-essential gene ${ }^{38}$. A MCC $=0.40$ was obtained, indicating that the model has a certain predictive capability. Interestingly, a value of 0.69 has been reported for a similar achievable value.

\begin{tabular}{|l|l|l|}
\hline Predicted & \multicolumn{2}{|c|}{ Experimental } \\
\hline & Essential & Non-essential \\
\hline Essential & $\mathrm{TP}=110$ & $\mathrm{FP}=76$ \\
\hline Non-essential & $\mathrm{FN}=69$ & $\mathrm{TN}=277$ \\
\hline
\end{tabular}




\section{DISCUSSION}

337 The successful application of TIS to C. autoethanogenum has provided a wealth of

338 information on gene essentiality in this industrially important acetogen and represents the

339 most thorough analysis of its kind performed to date in clostridia. The essentiality status of all

340 C. autoethanogenum genes can now be consulted (in Supplementary Table S1) before

341 directed knockouts are made. The conditionally essential genes are particularly useful when

342 hypothesising on gene functions and may be the starting point for further research with the 30

343 hypothetical proteins which were not required on rich medium, but were essential for

344 minimal medium autotrophic growth being the most obvious examples.

345 Essentiality in the Wood-Ljungdahl pathway (WLP). The generation of the methyl group

346 from $\mathrm{CO}$ first requires its oxidation to $\mathrm{CO}_{2}$ by a $\mathrm{CODH}$. C. autoethanogenum possesses three

347 such enzymes genome, namely CLAU_1578/CLAU_1579 (acsA), CLAU_2924

348 (CAETHG_3005) (cooS1) and CLAU_3807 (CAETHG_3899) (cooS2) ${ }^{17}$. Their independent

349 interruption via ClosTron mutagenesis ${ }^{41}$ showed that autotrophic growth was only abolished

350 in the acsA knockout mutant suggesting it is the only CODH required for growth on either

$351 \mathrm{CO}$ or $\mathrm{CO}_{2}$. Our data validates this conclusion with only acs $A$ being predicted as required for

352 growth on $\mathrm{CO}$ but not on YTF. The remaining putative $\mathrm{CODH}$ genes were required in neither

353 condition and there was no substantial change in insertion index between YTF and CO

354 conditions. The conditionally essential CODH encoded by acsA has an internally translated

355 stop codon (TGA) not found in the equivalent genes of related organisms and can

356 alternatively be thought of as two ORFs (CLAU_1578 and CLAU_1579) although it appears

357 that CLAU_1579 makes no separate product. It has been demonstrated that acsA can be

358 translated either as a $44 \mathrm{kDa}$ protein or as a $69 \mathrm{kDa}$ protein depending on whether the TGA

359 internal stop codon is the end of translation or whether it causes the incorporation of a 
selenocysteine residue ${ }^{23}$. It appears from our data that both ORFs are required under autotrophic conditions. Thus, the $44 \mathrm{kDa}$ protein alone does not appear to be sufficient for autotrophy and cells apparently require the $69 \mathrm{kDa}$ protein to be autotrophic.

There are three putative formate dehydrogenases in the $C$. autoethanogenum genome encoded by CLAU_0081, CLAU_2712/CLAU_2713 $(f d h A)$, and CLAU_2907. Of these, $f d h A$ alone appears to be essential only on $\mathrm{CO}$ while the remaining two genes are required in neither tested condition. The most important formate dehydrogenase is therefore $f d h A$ which is found in a complex with an NADP-specific electron-bifurcating [FeFe]-hydrogenase (Hyt) ${ }^{42}$. Two of the three putative formate dehydrogenases are selenoenzymes which may be higher efficiency than the cysteine-containing analogues, it is therefore tempting to speculate that the non-selenoenzyme formate dehydrogenase may be present as a backup for low selenium conditions ${ }^{43}$. However, it appears from our data that neither CLAU_0081 nor CLAU_2907 could provide sufficient activity in the $f d h A$ mutants for them to not be outcompeted causing $f d h A$ to appear essential under autotrophic conditions.

374 The steps from formate to methyl-THF are catalysed by the products of CLAU_1572-

375 CLAU_1576 which all appear to be required for growth on CO. CLAU_1574 and

376 CLAU_1576 additionally appear to also be required for growth on the rich medium.

377 The methyl group of methyl-THF is transferred to the Corrinoid Iron-Sulfur Protein

378 (CoFeSp) cofactor before being combined with the carbonyl group supplied by another

379 molecule of CO by the action of the ACS (acetyl-CoA synthase). The ACS is encoded by the region CLAU_1566-70 in which CLAU_1566, CLAU_1568, and CLAU_1569 were only required on CO whereas CLAU_1567 and CLAU_1570 were required on CO and on YTF. 
382 Essentiality in the metabolism from acetyl-CoA. There are four main carbon compounds

383

384

385

386

387

388

389

390

391

392

393

394

395

396

397

398

at the end points of metabolism for $C$. autoethanogenum: acetate, ethanol, 2,3 - butanediol, and lactate. The route to acetate from acetyl-CoA proceeds through acetyl phosphate; catalysed by the enzymes phosphate acetyltransferase (encoded by pta) and acetate kinase (encoded by $a c k A$ ). Both pta and $a c k A$ were found to be essential when growing on rich medium. The step from acetyl phosphate to acetate regenerates ATP and so this pathway may be required for energy generation.

However, pta has been knocked out in C. ljungdahlii ${ }^{44-46}$ where it significantly impaired growth rates and acetate formation. The $C$. ljungdahlii pta knockout may be viable only because of a second putative phosphate acetyltransferase (WP_063556670.1) which is annotated as a bifunctional enoyl-CoA hydratase/phosphate acetyltransferase and has no homolog in C. autoethanogenum. The bifunctional enoyl-CoA hydratase/phosphate acetyltransferase may be producing sufficient ATP for cells to be viable. The absence of an alternative phosphate acetyltransferase in C. autoethanogenum is likely the cause of the essentiality of pta in our data.

The route to ethanol can proceed from acetyl-CoA either straight to acetaldehyde and then to ethanol or via acetate, then acetaldehyde and finally ethanol. The more direct route from acetyl-CoA to acetaldehyde is catalysed by an acetaldehyde dehydrogenase (EC 1.2.1.10) which could be encoded by an estimated five genes within the $C$. autoethanogenum genome (CLAU_1772, CLAU_1783, CLAU_3204, CLAU_3655, CLAU_3656) none of which appear to be required in either growth condition. This could represent redundancy between these genes which further knockout studies could aim to confirm, or it could be that this route to ethanol is not required. The alternative route to ethanol via acetate is similar in that there are two predicted genes (CLAU_0089 and CLAU_0099) which could encode an aldehyde 
ferredoxin oxidoreductase (AOR; EC 1.2.7.5) but neither of them appear to be essential in

407 either growth condition. In case this the result is best explained by a lack of biological still viable autrophically ${ }^{23}$.

410 There are two candidate genes encoding pyruvate synthase enzymes for formation of pyruvate from acetyl-CoA (CLAU_0896 and CLAU_2947) of which only CLAU_2947 functions for the pathways leading to lactate and 2,3-butanediol appear non-essential. In the

414 case of the conversion of acetolactate to acetoin and in the production of lactate utilising

415 NADH there appears to be only one gene encoding the relevant functions (CLAU_2851 and

416 CLAU_1108 respectively); in these cases redundancy is unlikely to be the reason for their

417 non-essential status meaning it is more likely these are unnecessary biological routes.

418 Overall, our findings highlight that TIS represents a powerful functional genomics tool which 419 can be applied to less genetically tractable organisms using the methods applied here.

420 Presented data allows a confident determination of the Wood-Ljungdahl pathway genes of $C$. autoethanogenum and opens up future avenues of investigation into the genes which are essential for autotrophic growth with no obvious reason as to why.

\section{MATERIALS AND METHODS}

425 Microbiology. E. coli DH5alpha (NEB) was used for all for cloning and sExpress ${ }^{47}$ as a

426 conjugal donor. Strains were cultured at $37^{\circ} \mathrm{C}$ in LB broth with appropriate antibiotic

427 supplementation and 5-FC in a shaking incubator or on LB agar in a static incubator. $C$.

428 autoethanogenum was cultured and manipulated in an anaerobic workstation (Don Whitley) 
with an atmosphere of $80 \%$ nitrogen, $10 \%$ carbon dioxide and $10 \%$ hydrogen at $37{ }^{\circ} \mathrm{C}$. The three media used, were YTF (Table S4-S7), PETC (Table S8-S10) and Fermentation (Table detailed in Woods et al., $2019^{47}$. Briefly, this involved mixture of the donor and recipient cultures together and incubation on a non-selective YTF plate for $20 \mathrm{~h}$ before harvesting and plating onto selective YTF agar. Antibiotic selection for transposon plasmids was performed using chloramphenicol $(25 \mu \mathrm{g} / \mathrm{ml})$ and erythromycin $(500 \mu \mathrm{g} / \mathrm{ml})$ in E. coli or thiamphenicol used to select for the sExpress donor strain. D-cycloserine $(250 \mu \mathrm{g} / \mathrm{ml})$ was used to counterselect the sExpress donor strain. Fluorocytosine (FC) was supplemented at $30 \mu \mathrm{g} / \mathrm{ml}$ and IPTG at a concentration of $1 \mathrm{mM}$. Plasmid pMTL-CW20 maybe sourced from www.plasmidvectors.com.

DNA manipulations. Genomic DNA purifications were performed using bacterial gDNA extraction kits from Sigma Aldrich. Plasmid DNA was purified with mini-prep kits from NEB. Screening PCRs were performed using DreamTaq polymerase (Thermo Fisher).

444 Oligonucleotides were synthesised by Sigma Aldrich. Sanger sequencing was performed by Source Bioscience.

Mutant generation using CRISPR. A CRISPR in-frame deletion vector was designed as

447 previously described using the pMTL40000 CRISPR vector series ${ }^{48}$. In this case we employ

448 the trCas9 nickase variant under control of the $f d x$ promoter from $C$. sporogenes, a unique sgRNA under control of the constitutive $\operatorname{araE}$ promoter from C. acetobutylicum targeting $n f n$, and a homologous recombination cassette to allow the precise in-frame deletion of $n f n$.

451 Following vector assembly, the construct was transferred to wild type C. autoethanogenum

452 by conjugation using sExpress as the E. coli donor strain ${ }^{47}$. Following two rounds of 
selection on thiamphenicol and D-cycloserine, to select for recipient strains harbouring the

454 CRISPR vector and counterselect the E. coli donor cells, respectively, a colony PCR screen

455

456

457

458

459

460

461

462

463

464

465

466

467

468

469

470

471

472

473

474

475

476

was performed on resultant colonies, amplifying from the genomic locus flanking the regions

selected for homologous recombination. The screen revealed that the $n f n$ knockout mutant

was indeed present in the population, and the strain was sub-cultured for storage and

preparation of genomic DNA. Sanger sequencing from a high-fidelity PCR product

confirmed the precise in-frame deletion of $n f n$.

Assessment of transposon vectors. Transposon delivery vectors were transferred to $C$.

autoethanogenum via conjugal transfer from sExpress and selected for on YTF agar plates

supplemented with clarithromycin and D-cycloserine. Colonies were harvested from selection

plates by flooding with PBS and the entire cell suspension was serially diluted and spread

onto YTF agar plates supplemented with either clarithromycin and IPTG, thiamphenicol and

IPTG or clarithromycin to determine the transposition frequency and plasmid-retention in the presence of IPTG.

Transposon library creation. The transposon-delivery vector pMTL-CW20 was transformed into an E. coli conjugative donor strain sExpress ${ }^{47}$ which was used to transfer the plasmid into C. autoethanogenum $\mathrm{C} 24$. Twelve conjugations were performed simultaneously producing a total of around 81,000 transconjugant colonies on YTF agar supplemented with D-cycloserine and clarithromycin. All transconjugants were pooled and plated onto agar plates supplemented with IPTG, lactose and thiamphenicol to select for transposon mutants. Transposon mutant colonies were then harvested and inoculated to YTF broth supplemented with IPTG and thiamphenicol. The rich medium sequencing samples were taken from this liquid phase which was used to inoculate PETC pyruvate medium. The PETC pyruvate culture was allowed to reach stationary phase before being used as inoculum 
477 for the bioreactor where CO served as the carbon and energy source; a DNA sample was

478 taken from PETC pyruvate at the point of bioreactor inoculation. Samples were taken from

479 the bioreactor to check for the presence of pyruvate, monitor the OD and to serve as DNA

480 samples for identification of insertion sites.

481 Bioinformatics and metabolic modelling. Experimentally confirmed essential genes were

482 compared against metabolic modelling-derived essentiality. Lists of essential genes were

483 generated using the BioTraDIS toolkit approach as previous described ${ }^{49}$. A genome-scale

484 model (GSM) of CO-fed C. autoethanogenum was handled using the COBRA Toolbox in

485 MATLAB R2017b to predict gene essentiality ${ }^{36,50}$. Briefly, the wild type model was

486 subjected to Flux Balance Analysis (FBA) by selecting the maximization of the biomass yield

487 as the objective function ${ }^{37}$. Simulated single gene deletions provoking a reduction of at least

$4885 \%$ of the optimal specific growth rate of the wild type were deemed essential. Finally,

489 Matthew's correlation coefficient was used as a metric to assess the quality of the GSM

490 predictions, where " 1 " is a perfect correlation between experimental and predicted gene

491 essentiality, " 0 " no correlation, and " 1 " perfect anti-correlation ${ }^{39}$. The model and the scripts

492 are available in GitHub (https://github.com/SBRCNottingham/C.auto_essentiality). 
Sequencing and bioinformatics. Sequencing library preparation was performed as an amplicon

494 library using a splinkerette adapter ${ }^{49}$. Genomic DNA was fragmented to an average of $400 \mathrm{bp}$ at a ratio of $1.5 \mathrm{X}$ beads to sample. Fragmented DNA was end repaired and A-tailed using the

NEB Ultra II library preparation kit. Splinkerette adapters were ligated onto the end of A-tailed fragments with reagents from the Ultra II library preparation kit. A $1 \mathrm{X}$ bead purification was performed before an I-SceI digest step to cleave plasmid DNA between the library primer and P7 primer. Another $1 \mathrm{X}$ bead purification was performed before PCR amplification of the transposon junctions using KAPA HiFi polymerase. An initial denaturing step of $95^{\circ} \mathrm{C}$ for two

502 min was followed by 20 rounds of $95^{\circ} \mathrm{C}$ for $20 \mathrm{sec}, 61^{\circ} \mathrm{C}$ for $30 \mathrm{sec}$ then $72^{\circ} \mathrm{C}$ for $30 \mathrm{sec}$ before 503 a final extension of two min at $72^{\circ} \mathrm{C}$ was performed.

504 PCR products with a size range of 250-500 bp were gel extracted from a low-melt agarose gel

505 using the NEB monarch gel extraction kit. Gel extracted products were analysed on an

506 Agilent bioanalyser using a DNA 1000 chip and quantified via Qubit and qPCR. Two

507 separate runs were performed on an Illumina MiSeq.

508 Raw sequences were trimmed before filtering for reads which contain the expected

509 transposon tag. The transposon tag was removed from reads which could then be mapped to

510 the C. autoethanogenum genome to identify insertion sites. The BioTraDIS analysis pipeline 511 was used for these steps and for subsequent analysis ${ }^{49}$.

\section{ACKNOWLEDGEMENTS}

513 This work was funded by the Biotechnology and Biological Sciences Research Council [grant

514 numbers BB/L502030/1, BB/K00283X/1, BB/L013940/1]. NPM acknowledges funding from

515 LanzaTech as part of BB/L502030/1. The funders had no role in study design, data collection 
516 and analysis, decision to publish, or preparation of the manuscript. Sequencing was

517 performed by Deep Seq (University of Nottingham) with thanks to Nadine Holmes, Matthew

518 Carlile, and Victoria Wright.

520 Author Contributions

$521 \mathrm{CW}, \mathrm{CMH}$ and NPM planned the study. CW and CMH undertook the experimental work.

522 CTA performed the metabolic modelling. Data analysis was undertaken by CW. CW and

523 CMH drafted the manuscript. All authors reviewed, edited, and approved the final version of

524 the manuscript.

\section{Competing Interests}

526 MK and SDS are employees of LanzaTech, a for profit with commercial interest in clostridial 527 gas fermentation.

\section{REFERENCES}

530 1. Hutchison III CA, Chuang R-Y, Noskov VN, Assad-Garcia N, Deerinck TJ, Ellisman MH, Gill J, Kannan K, Karas BJ, Ma L, Pelletier JF, Qi Z-Q, Richter RA, Strychalski 351:6253. 

19:2308-2316.

3. Gawronski JD, Wong SMS, Giannoukos G, Ward DV, Akerley BJ. 2009. Tracking insertion mutants within libraries by deep sequencing and a genome-wide screen for Haemophilus genes required in the lung. Proc Natl Aca Sci USA 106:16422-16427.

4. Goodman AL, McNulty NP, Zhao Y, Leip D, Mitra RD, Lozupone CA, Knight R, Gordon JI. 2009. Identifying genetic determinants needed to establish a human gut symbiont in its habitat. Cell Host Microbe 6:279-89.

545 5. Chao MC, Abel S, Davis BM, Waldor MK. 2016. The design and analysis of transposon insertion sequencing experiments. Nat Rev Microbiol 14:119-128.\#

547 6. Dembek M, Barquist L, Boinett CJ, Cain AK, Mayho M, Lawley TD, Fairweather NF, Fagan RP. 2015. High-throughput analysis of gene essentiality and sporulation in Clostridium difficile. mBio. 6(2):e02383.

7. Ragsdale SW, Pierce E. 2008. Acetogenesis and the Wood-Ljungdahl pathway of $\mathrm{CO}_{2}$ fixation. Biochim Biophys Acta - Proteins Proteomics 1784:1873-1898. Schlegel H, Bowien B (ed), Autotrophic Bacteria. Science Tech Publishers, Madison.

554 9. Fast AG, Papoutsakis ET. 2012. Stoichiometric and energetic analyses of nonphotosynthetic $\mathrm{CO}_{2}$-fixation pathways to support synthetic biology strategies for production of fuels and chemicals. Curr Opin Chem Eng 1:380-395. 
10. Drake HL, Daniel SL. 2004. Physiology of the thermophilic acetogen Moorella thermoacetica. Res Microbiol 155:869-883.

11. Ragsdale SW. 2008. Enzymology of the Wood-Ljungdahl pathway of acetogenesis. Ann N Y Acad Sci 1125:129-136 doi: 10.1196

12. Shin J, Song Y, Jeong Y, Cho BK. 2016. Analysis of the core genome and pan-genome of autotrophic acetogenic bacteria. Front Microbiol 7:1531.

13. Müller V, Pal Chowdhury N, Basen M. 2018. Electron Bifurcation: A Long-Hidden Energy-Coupling Mechanism. Ann Rev Microbiol 72:331-353.

14. Köpke M, Simpson SD. 2020. Pollution to products: recycling of 'above ground' carbon by gas fermentation. Curr Opin Biotechnol 65:180-189.

15. Köpke M, Simpson S, Liew F. Chen W. 2012. Fermentation Process For Producing i sopropanol Using a Recombinant Microorganism. US patent 936,586,8B2.

16. Humphreys CM, McLean S, Schatschneider S, Millat T, Henstra AM, Annan FJ, Breitkopf R, Pander B, Piatek P, Rowe P, Wichlacz AT, Woods C, Norman R, Blom J, Goesman A, Hodgman C, Barrett D, Thomas NR, Winzer K, Minton NP. 2015. Whole genome sequence and manual annotation of Clostridium autoethanogenum, an industrially relevant bacterium. BMC Genomics 16:1-10.

17. Brown SD, Nagaraju S, Utturkar S, De Tissera S, Segovia S, Mitche W, Land ML, Dassanayake A, Köpke M. 2014. Comparison of single-molecule sequencing and hybrid approaches for finishing the genome of Clostridium autoethanogenum and analysis of CRISPR systems in industrial relevant Clostridia. Biotechnol Biofuels 7:40. 
18. Marcellin E. Behrendorff JB, Nagaraju S, DeTissera S, Segovia S, Palfreyman RW, Daniell J, Licona-Cassani C, Quek L, Speight R, Hodson MP, Simpson SD, Mitchell WP, Köpke M, Nielsena LK. 2016. Low carbon fuels and commodity chemicals from waste gases-systematic approach to understand energy metabolism in a model acetogen. Green Chem 18:3020-3028.

19. Minton NP, Ehsaan M, Humphreys CM, Little GT, Baker J, Henstra AM, Liew F, Kelly ML, Sheng L, Schwarz K, Zhang Y. 2016. A roadmap for gene system development in Clostridium. Anaerobe 41:104-112.

20. Banerjee A, Leang C, Ueki T, Nevin KP, Lovley, DR. 2014. Lactose-inducible system for metabolic engineering of Clostridium ljungdahlii. Appl Environ Microbiol 80:2410-2416.

21. Heap JT, Ehsaan M, Cooksley CM, Ng YK, Cartman ST, Winzer K, Minton NP. 2012. Integration of DNA into bacterial chromosomes from plasmids without a counterselection marker. Nucleic Acids Res $\mathbf{4 0}$ doi:10.1093

22. Ng YK, Ehsaan M, Philip S, Collery MM, Janoir C, Collignon A, Cartman ST, Minton NP. 2013. expanding the repertoire of gene tools for precise manipulation of the Clostridium difficile genome: allelic exchange using pyrE alleles. PLoS One 8:e56051

23. Liew F, Henstra AM, Köpke M, Winzer K, Simpson SD, Minton NP. 2017. Metabolic engineering of Clostridium autoethanogenum for selective alcohol production. Metab Eng 40:104-114. 
600

601

602

603

604

605

606

607

608

609

610

611

612

613

614

615

616

617

618

619

24. Zhang Y, Grosse-Honebrink A, Minton NP. 2015. A Universal Mariner Transposon System for Forward Genetic Studies in the Genus Clostridium. PLoS One 10:e0122411.

25. Dempwolff F, Sanchez S, Kearns DB. 2020. TnFLX: A third-generation mariner-based transposon system for Bacillus subtilis. Appl Environ Microbiol. 86:e02893-19

26. Bossé JT, Li Y, Leanse LG, Zhou L, Chaudhuri RR, Peters SE, Wang J, Maglennon GA, Holden MTG, Maskell DJ, Tucker AW, Wren BW, Rycroft AN, Langford PR. 2018. Rationally designed mariner vectors to allow functional genomic analysis of Actinobacillus pleuropneumoniae and other bacteria by transposon-directed insertionsite sequencing (TraDIS). bioRxiv $\mathbf{4 3 3 0 8 6}$

27. Heap JT, Pennington OJ, Cartman ST, Minton NP. 2009. A modular system for Clostridium shuttle plasmids. J Microbiol Methods 78:79-85.

28. Purdy D, O'Keeffe TA, Elmore M, Herbert M, McLeod A, Bokori-Brown M, Ostrowski A, Minton NP. 2002. Conjugative transfer of clostridial shuttle vectors from Escherichia coli to Clostridium difficile through circumvention of the restriction barrier. Mol Microbiol 46:439-452.

29. Lampe DJ, Akerley BJ, Rubin EJ, Mekalanos JJ, Robertson HM. 1999. Hyperactive transposase mutants of the Himar1 mariner transposon. Proc Natl Acad Sci USA 96: $11428-11433$.

30. Iwasaki W, Sekine S, Kuroishi C, Kuramitsu S, Shirouzu M, Yokoyama S. 2006. Structural Basis of the Water-assisted Asparagine Recognition by Asparaginyl-tRNA Synthetase. J Mol Biol 360:329-342. 
31. Schuchmann K, Müller V. 2014. Autotrophy at the thermodynamic limit of life: A model for energy conservation in acetogenic bacteria. Nat Rev Microbiol 12:809-821.

32. Köpke M, Held C, Hujer S, Liesegang H, Wiezer A, Wollherr A, Ehrenreich A, Liebl W, Gottschalk G, Dürre P. 2010. Clostridium ljungdahlii represents a microbial production platform based on syngas. Proc Natl Acad Sci 107:13087-13092.

33. Tremblay P, Zhang T, Dar SA, Leang C, Lovely DR. 2012. The Rnf complex of Clostridium ljungdahlii is a proton-translocating NAD+ oxidoreductase essential for autotrophic growth. mBio $4: 1-8$.

34. Westphal L, Wiechmann A, Baker J, Minton N P, Müller V. 2018. The Rnf complex is an energy-coupled transhydrogenase essential to reversibly link cellular NADH and ferredoxin pools in the acetogen Acetobacterium woodii. J Bacteriol 200:e0357-18.

35. Suharti S, Wang M, de Vries S, Ferry JG. 2014. Characterization of the RnfB and RnfG Subunits of the Rnf Complex from the Archaeon Methanosarcina acetivorans. PLoS One 9:e97966.

36. Norman ROJ, Millat T, Schatschneider S, Henstra AM, Breitkopf R, Pander B, Annan FJ, Piatek P, Hartman HB, Poolman MG, Fell DA, Winzer K, Minton NP, Hodgman C. 2019. Genome-scale model of C. autoethanogenum reveals optimal bioprocess conditions for high-value chemical production from carbon monoxide. Eng Biol 3:32-40.

37. Orth JD, Thiele I, Palsson BO. 2010. What is flux balance analysis? Nat Biotechnol 28:245-248. 
641

642

643

644

645

646

647

648

649

650

651

652

653

654

655

656

657

658

659

660

661

662

38. Chicco D, Tötsch N, Jurman G. 2021. The Matthews correlation coefficient (MCC) is more reliable than balanced accuracy, bookmaker informedness, and markedness in two-class confusion matrix evaluation. BioData Min 14:13.

39. Salvy P, Hatzimanikatis V. 2020. The ETFL formulation allows multi-omics integration in thermodynamics-compliant metabolism and expression models. Nat Commun 11:1-17.

40. Orth JD, Conrad TM, Na J, Lerman JA, Nam H, Feist AM, Palsson BØ. 2011. A comprehensive genome-scale reconstruction of Escherichia coli metabolism-2011. Mol Syst Biol 7:535.

41 Liew FM, Martin ME, Tappel RC, Heijstra BD, Mihalcea C, Köpke M. 2016. Gas Fermentation-A flexible platform for commercial scale production of low-carbon-fuels and chemicals from waste and renewable feedstocks. Front Microbiol 7 doi:10.3389.

42. Wang S, Huang H, Kahnt J, Mueller AP, Köpke M, Thauer RK. 2013. NADP-Specific electron-bifurcating [FeFe]-hydrogenase in a functional complex with formate dehydrogenase in Clostridium autoethanogenum grown on CO. J Bacteriol 195:43734386

43. Köpke M, Mihalcea C, Liew F, Tizard JH, Ali MS, Conolly JJ, Al-Sinawi B, Simpson SD. 2011. 2,3-Butanediol production by acetogenic bacteria, an alternative route to chemical synthesis, using industrial waste gas. Appl Environ Microbiol 77:5467-5475.

44. Huang H, Chai C, Li N, Rowe P, Minton NP, Yang S, Jiang W, Gu Y. 2016. CRISPR/Cas9-Based Efficient Genome Editing in Clostridium ljungdahlii, an Autotrophic Gas-Fermenting Bacterium. ACS Synth Biol 5:1355-1361. 
663

664

665

666

667

668

669

670

671

672

673

674

675

676

677

678

679

680

681

682

683

684

45. Latif H, Zeidan AA, Nielsen AT, Zengler K. 2014. Trash to treasure: Production of biofuels and commodity chemicals via syngas fermenting microorganisms. Curr Opin Biotechnol 27:79-87.

46. Lo J, Humphreys JR, Jack J, Urban C, Magnusson L, Xiong W, Gu Y, Ren ZJ, Maness PC. 2020. The Metabolism of Clostridium ljungdahlii in Phosphotransacetylase Negative Strains and Development of an Ethanologenic Strain. Front Bioeng Biotechnol 8:560726.

47. Woods C, Humphreys CM, Rodrigues RM, Ingle P, Rowe P, Henstra AM, Köpke M, Simpson SD, Winzer K, Minton NP. 2019. A novel conjugal donor strain for improved DNA transfer into Clostridium spp. Anaerobe 59:184-191.

48. Ingle P, Groothuis D, Rowe P, Huang H, Cockayne A, Kuehne SA, Jiang W, Gu Y, Humphreys CM, Minton NP. 2019. Generation of a fully erythromycin-sensitive strain of Clostridioides difficile using a novel CRISPR-Cas9 genome editing system. Sci Rep 9:8123.

49. Barquist L, Mayho M, Cummins C, Cain AK, Boinett CJ, Page AJ, Langridge GC, Quail MA, Keane JA, Parkhill J. 2016. The TraDIS toolkit: Sequencing and analysis for dense transposon mutant libraries. Bioinformatics 32:1109-1111.

50. Schellenberger J, Que R, Fleming RM, Thiele I, Orth JD, Feist AM, Zielinski DC, Bordbar A, Lewis NE, Rahmanian S, Kang J, Hyduke DR, Palsson BØ. 2011. Quantitative prediction of cellular metabolism with constraint-based models: The COBRA Toolbox v2.0. Nat Protoc 6:1290-1307. 